\title{
Alteplase-Tenecteplase Trial Evaluation for Stroke Thrombolysis (ATTEST 2)
}

\author{
A. Murray', K. Muir'1, I. Ford1', J. Wardlaw² , G.Ford ${ }^{3}$
}

${ }^{1}$ University of Glasgow, ${ }^{2}$ University of Edinburgh, ${ }^{3}$ Oxford University Hospitals NHS Trust

\section{Background and purpose}

Intravenous (IV) thrombolysis with alteplase is the only medical treatment currently approved for acute ischaemic stroke. Dose and treatment schedule remain those established in the NINDS trial in 1995. Alteplase significantly improves the likelihood of good outcomes, but efficacy is suboptimal in large artery occlusion.

There is potentially substantial benefit from better IV thrombolytic agents. Newer plasminogen activators have improved pharmacological characteristics (longer half-life, higher fibrin affinity, less disruption to endogenous haemostasis). Based on data from selected patients with large artery occlusion, and individual patient data meta-analysis, we hypothesise that tenecteplase will be superior to alteplase. Data from EXTEND-IA TNK provides further strong support for this hypothesis but was restricted to selected patients with large artery occlusion. Substantially more data are required to establish the true risk-benefit profile compared with alteplase in the general stroke population.

\section{Trial design}

ATTEST 2 is a prospective, randomised controlled multicentre parallel group trial comparing IV rtPA with IV tenecteplase in patients eligible for IV thrombolysis within guidelines (currently $<4.5 \mathrm{~h}$ after onset, with no upper age limit) based on non-contrast CT imaging. The trial uses a PROBE (Prospective Randomised Open Blinded End-point) design.

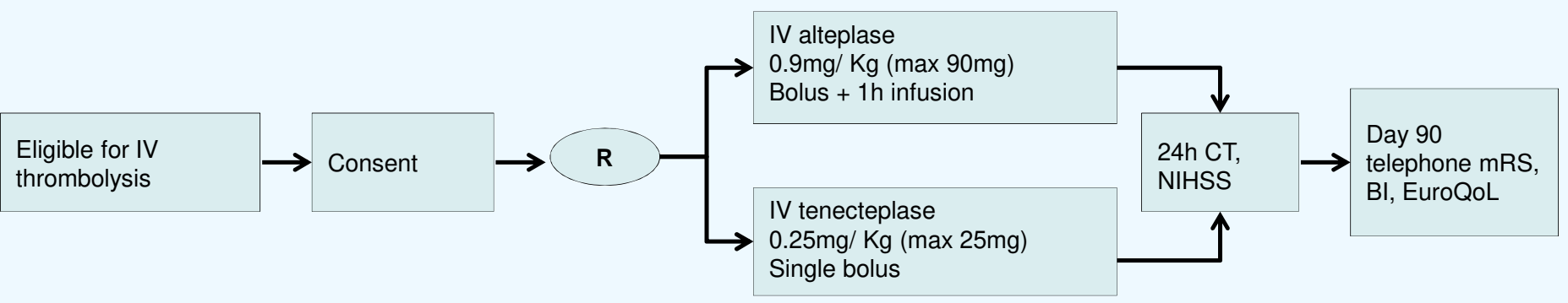

\section{Inclusion criteria}

$\checkmark$ Eligible for IV thrombolysis.

$\checkmark$ Male or non-pregnant female $\geq 18$ years of age.

$\checkmark<4.5 \mathrm{~h}$ after symptom onset.

$\checkmark$ Consent of patient or legal representative.

$\checkmark$ Independent prior to the stroke (estimated mRS 0-1).

\section{Exclusion criteria}

$>$ Contraindications to thrombolytic therapy (see protocol for further details).

$>$ Acute endovascular treatment for stroke planned.

$>$ Any major medical condition likely to limit survival to day 90 .

$>$ Unavailable for day 90 follow-up.

\section{Study endpoints}

\begin{tabular}{|c|c|c|}
\hline Primary Outcome: & mRS at 90 days (ordinal shift analysis) & \\
\hline \multirow[t]{3}{*}{ Secondary Outcomes: } & Full neurological recovery (mRS 0-1 versus 2-6). & Independent recovery (mRS score 0-2 versus 3-6). \\
\hline & $\begin{array}{l}\text { Early major neurological improvement of } 8 \text { or more } \\
\text { points, or return to NIHSS total score of } 0 \text { or } 1 \text {, at } 24 \\
\text { hours. }\end{array}$ & Health Related Quality of Life (EQ-5D) at day 90. \\
\hline & Barthel Index scores at day 90. & Need for thrombectomy. \\
\hline
\end{tabular}

In addition there are several safety endpoints (including mortality and symptomatic intracranial haemorrhage rates).

\section{Study status}

- Regulatory and ethical approvals Q3 2016.

- 14 centres currently active, with 112 patients randomised to date.

- Approx. 60 UK centres will recruit 1870 patients.

\section{Conclusion}

An agent with superior risk:benefit ratio compared to alteplase would improve outcomes and may reduce the need for thrombectomy. Even for equivalent outcomes, superior ease of use of tenecteplase would confer significant clinical advantages.

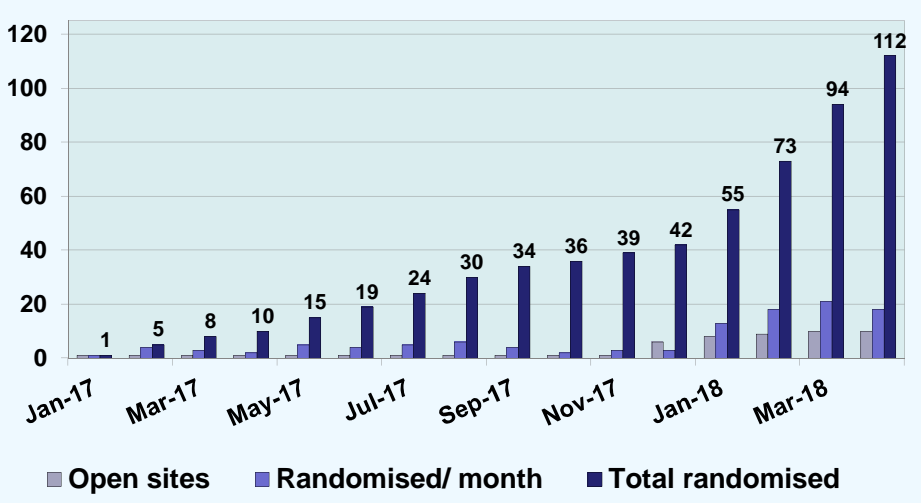

\section{Contacts \& acknowledgements}

Cl: Prof Keith Muir (Keith.Muir@glasgow.ac.uk); Trial manager: Dr Alicia Murray (Alicia.Murray@glasgow.ac.uk) This study is funded by the Stroke Association \& British Heart Foundation. 